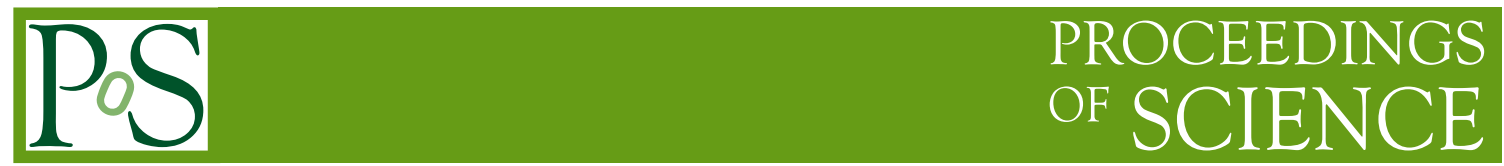

\title{
Physics with photons in ALICE
}

\author{
Yuri Kharlov* \\ Institute for High Energy Physics, Protvino, 142281, Russia \\ E-mail: Yuri.Kharlov@cern.ch
}

\section{Lamia Benhabib}

SUBATECH, 4 rue Alfred Kastler, BP 2072244307 Nantes CEDEX 3, France

\section{Renzhuo Wan}

IOPP-CCNU, Wuhan, China

\section{(for the ALICE collaboration)}

The ALICE experiment at LHC was designed and built to study properties of a hot quark matter produced in heavy ion collisions at $\sqrt{s_{N N}}=5.5 \mathrm{TeV}$. This paper describes capability of the ALICE to study photon physics with PHOS, EMCAL and PMD detectors. The ALICE potential is illustrated by predictions for direct photon and neutral mesons spectra to be measured in the first LHC run with proton-proton collisions in the PHOS detector.

2008 Physics at LHC

September 29 - 4 October 2008

Split, Croatia

${ }^{*}$ Speaker. 


\section{Introduction}

ALICE [1], a dedicated heavy-ion experiment at LHC, is equipped by a set of photon detectors which will detect photons in a wide kinematic range.

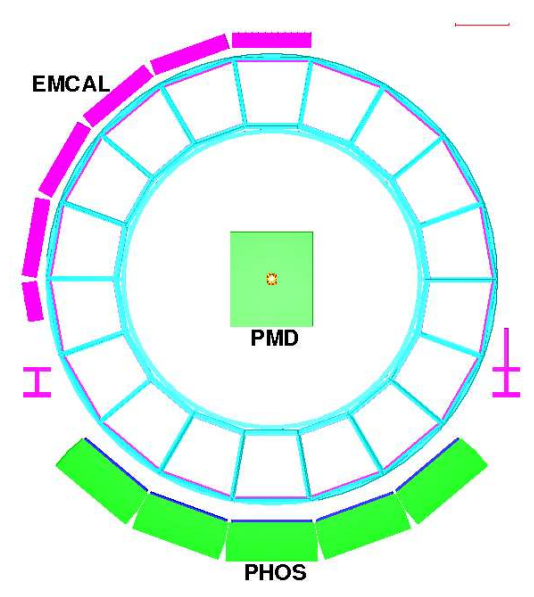

Figure 1: ALICE setup with photon detectors PHOS, EMCAL and PMD

The most precise detector, the photon spectrometer (PHOS) [2] is designed to measure inclusive direct photon and neutral meson spectra at transverse momenta from 0.5 to 100 $\mathrm{GeV} / c$ in an azimuth angle range $\Delta \phi=100^{\circ}$ and rapidity range $|y|<0.12$. The PHOS has a fine granularity, $\Delta \phi \times$ $\Delta y=4.8 \cdot 10^{-3} \times 4.3 \cdot 10^{-3}$ which allows to separate showers at a high-multiplicity environment. A large-aperture electromagnetic calorimeter (EMCAL) [3] will cover $\Delta \phi=$ $110^{\circ}$ and $|y|<0.7$ and will be used to measure a neutral jet component by detecting photons up to $p_{T}<250 \mathrm{GeV} / \mathrm{c}$. The EMCAL granularity $\Delta \phi \times \Delta y=15 \cdot 10^{-3} \times 15 \cdot 10^{-3}$ is optimized for jet identification. The photon multiplicity detector (PMD) [4] is a preshower detector of $2.7 X_{0}$ thickness. It will measure the multiplicity and spatial distribution of photons on an event-by-event basis in order to study event shapes and fluctuations in the forward region $2.3<y<3.5$. By the LHC start, the ALICE will have 3/5 of PHOS, 1/10 of EMCAL and 2/3 of PMD modules.

\section{Physics objectives}

The main source of photons in proton-proton and heavy ion collisions are decay photons from neutral mesons, like $\pi^{0} \rightarrow \gamma \gamma, \eta \rightarrow \gamma \gamma, \omega \rightarrow \pi^{0} \gamma$, etc. Measurement of inclusive spectra for these mesons in electromagnetic calorimeters is much easier than those for charged hadrons reconstructed in a tracking system. For example, the $\pi^{0}$ spectrum can be measured with the detector PHOS in a wide range of transverse momenta, $1<p_{T}<50 \mathrm{GeV} / \mathrm{c}$ using an invariant-mass analysis, and up to $p_{T}<80 \mathrm{GeV} / \mathrm{c}$ using a shower shape analysis [5]. Spectra of neutral mesons in $p p$ collision is an appropriate tool for validating $\mathrm{pQCD}$ predictions, while these spectra in $p A$ and $A A$ collisions are a good probe for the parton energy loss in cold and hot nuclear matter, via the nuclear modification factor $R_{A A}$, see [6].

Direct photons can be produced in several processes [7]. In $p p$ and $A A$ collisions, the photons are produced in hard QCD processes with two initial-state partons of the colliding nuclei. The next-to-leading order QCD processes can produce photons in gluon and quark fragmentation, these photons are referred to as fragmentation photons. In $A A$ collisions the photons are emitted off rescattering of thermilized quarks and gluons in a hot QGP, or off hadrons in a cooled hadronic gas. Such photons are referred to as thermal photons.

Jet detection in $p p$ and $A A$ collisions is also considered as an important hard probe of QGP properties. The neutral component of jets, constituted mainly of neutral mesons, is a significant contribution to the total jet energy, and the detector EMCAL of the ALICE experiment, together with the ALICE central tracking system, is designed for a good jet identification. 
As it has been proven by the heavy-ion experiments at RHIC, correlation functions are rather sensitive for various nuclear effects, and allow to study properties of a quark matter more extensively. Photon-hadron and photon-jet correlations measured in the ALICE with the PHOS and EMCAL detectors will reveal the properties of a parton energy loss in hot nuclear matter.

\section{Photon observables in PHOS in pp collisions}

The ALICE experiment will take data from the first LHC run with proton-proton collisions. From the point of view of ultra-relativistic nuclear physics, these $p p$-data are needed as a reference point for heavy-ion collisions, because nuclear modification effects will be compared with cold nuclear matter produced in $p p$ collisions, scaled by a number of binary collisions. However, photon observables measured in $p p$ collisions are interesting irrespectively to heavy ion collisions. Inclusive spectra of direct photons and neutral mesons detected in their photon decay channels are served as probes of perturbative QCD.

Here we give predictions for cross sections of direct photons, $\pi^{0}, \eta$ and $\omega(782)$ mesons in $p p$ collisions at LHC energies and estimate their detection rates in the PHOS detector in the first LHC run. Although the first LHC collision energy is anticipated to be equal to $10 \mathrm{TeV}$, for completeness the cross sections were calculated for different possible LHC energies. The cross section of direct photons and $\pi^{0}$ mesons were calculated in next-to-leading order pQCD using the program INCNLO v.1.5 [8] with a PDF set CTEQ5M and fragmentation functions KKP for $\pi^{0}$ and BGF set II for direct photons. These cross sections are shown in Fig.2. Cross sections of $\eta$ and $\omega(782)$ mesons
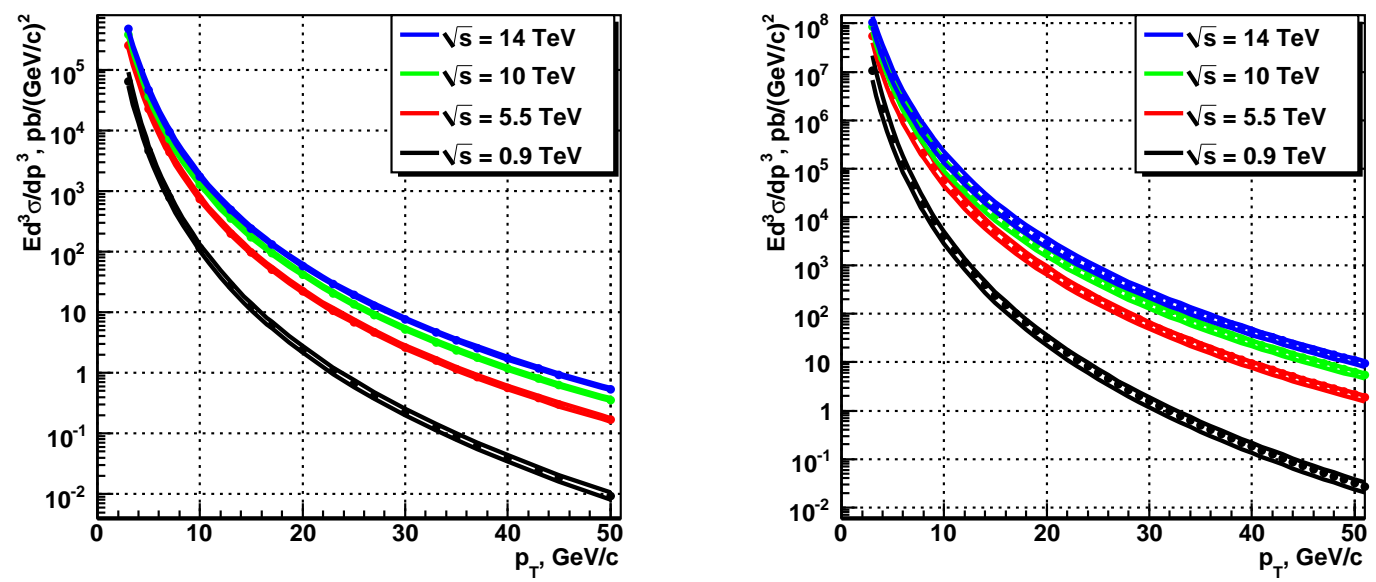

Figure 2: Production cross sections of direct photons (left) and $\pi^{0}$ (right) in $p p$ collisions at $0.9,5.5,10$ and $14 \mathrm{TeV}$.

were obtained in leading order pQCD by the event generator Pythia v.6.214 [9], see Fig.3.

Having obtained cross sections $d \sigma / d p_{T}$, the number of detected events $d N / d p T$ can be estimated for the first LHC run:

$$
\frac{d N}{d p_{T}}=\frac{d \sigma}{d p_{T}} \cdot \mathscr{L} \cdot T \cdot A \cdot \varepsilon,
$$

where $\mathscr{L}$ is a luminosity, $T$ is a duration of data taking, $A$ is the acceptance, and $\varepsilon$ includes various corrections to a detected spectrum due to reconstruction and trigger efficiency, off-vertex background and photon conversion in the ALICE medium. 

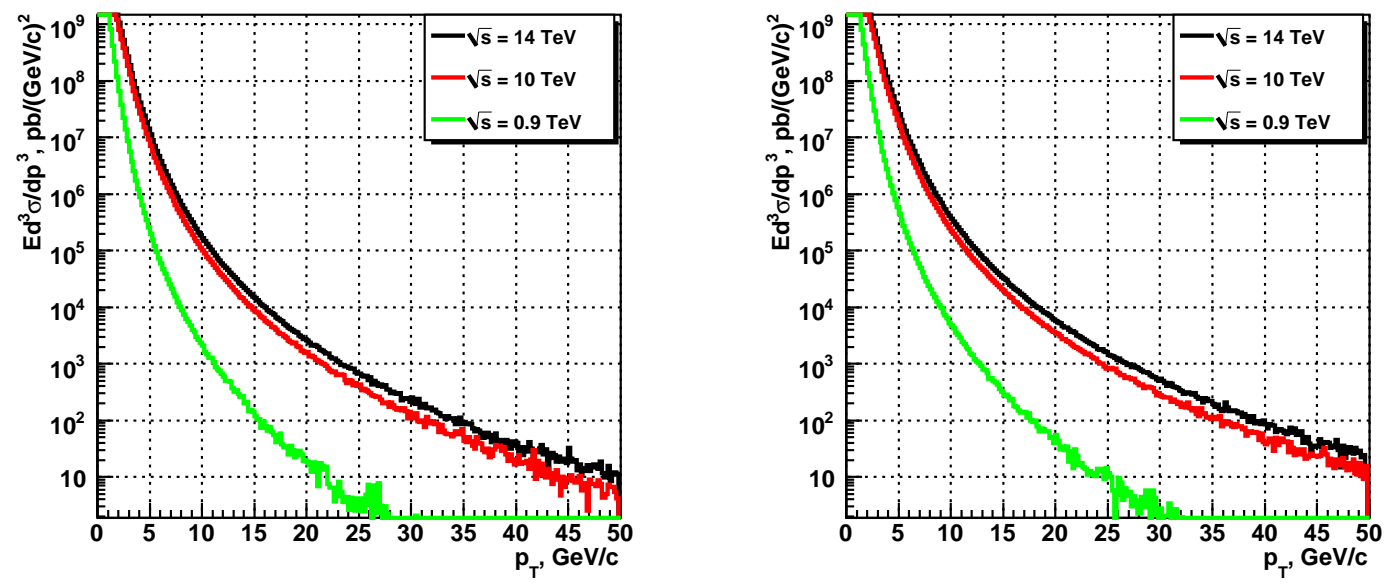

Figure 3: Production cross sections of $\eta$ (left) and $\omega$ (782) (right) in $p p$ collisions at $0.9,5.5,10$ and $14 \mathrm{TeV}$.

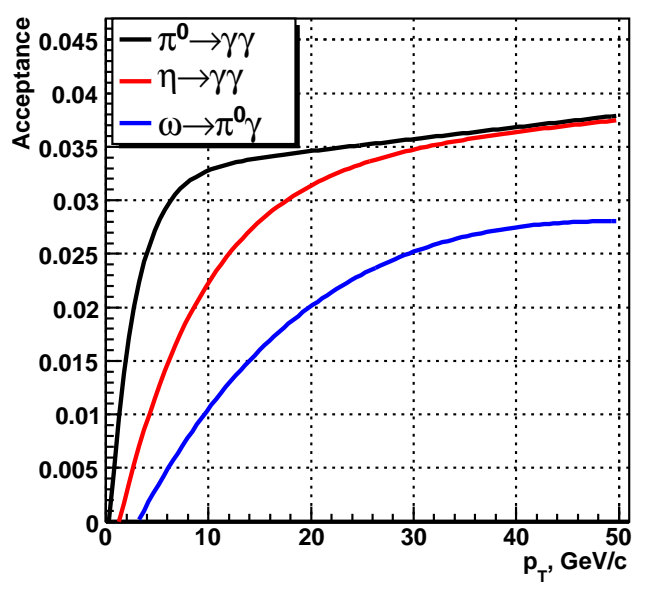

Figure 4: Acceptance of $\pi^{0} \rightarrow \gamma \gamma, \eta \rightarrow \gamma \gamma$ and $\omega \rightarrow \pi^{0} \gamma \rightarrow 3 \gamma$ in 3 PHOS modules.

We assume several scenarios of the first LHC run with proton beams. The collision energy of proton-proton beams was taken as $10 \mathrm{TeV}$. Accumulated statistics can be expressed via the integral luminosity $\int \mathscr{L} d T$ equal to $10 \mathrm{nb}^{-1}, 100 \mathrm{nb}^{-1}$ or $300 \mathrm{nb}^{-1}$, which can be achieved at the luminosity $\mathscr{L}=5 \cdot 10^{28} \mathrm{~cm}^{-2} \mathrm{~s}^{-1}$ and a run time of 3 days, 30 days or 3 months, respectively. Geometrical acceptances $A$ for direct photons is solely defined by the aperture of the PHOS detector, and independent on $p_{T}$. Acceptances for $\pi^{0}$ and $\eta$ mesons were calculated in their 2-photon decay channel, and for $\omega$ in the $\pi^{0} \gamma$ decay channel with a consequent decay of $\pi^{0} \rightarrow 2 \gamma$. Acceptances for 3 PHOS modules are shown in Fig.4. Other correction factors $\varepsilon$ for $\pi^{0}, \eta$ and $\omega$ were estimated as a product of single-photon reconstruction efficiencies $\varepsilon_{\gamma}=0.85$ (see [5]). The number of detected direct photons and neutral mesons in photon decay channels are shown in Fig. 5 for three values of integrated luminosities.

\section{Conclusion}

Photon observables constitute an important contribution to the physics program of the ALICE experiment. The first LHC run with $p p$ collisions at $\sqrt{s}=10 \mathrm{TeV}$ will produce enough data to measure inclusive spectra of neutral mesons and direct photons. Depending on LHC run scenarios, these spectra can be measured for $\pi^{0}$ at $p_{T}<25-50 \mathrm{GeV} / \mathrm{c}, \eta$ at $p_{T}<20-40 \mathrm{GeV} / \mathrm{c}, \omega(782)$ at $p_{T}<15-30 \mathrm{GeV} / \mathrm{c}$, and direct photons at $p_{T}<10-20 \mathrm{GeV} / \mathrm{c}$. These spectra will allow to validate $\mathrm{pQCD}$ calculations in $p p$ collisions and provide reference data for $A A$ collisions.

The work was partially supported by the INTAS grant $05-103-7484$. 

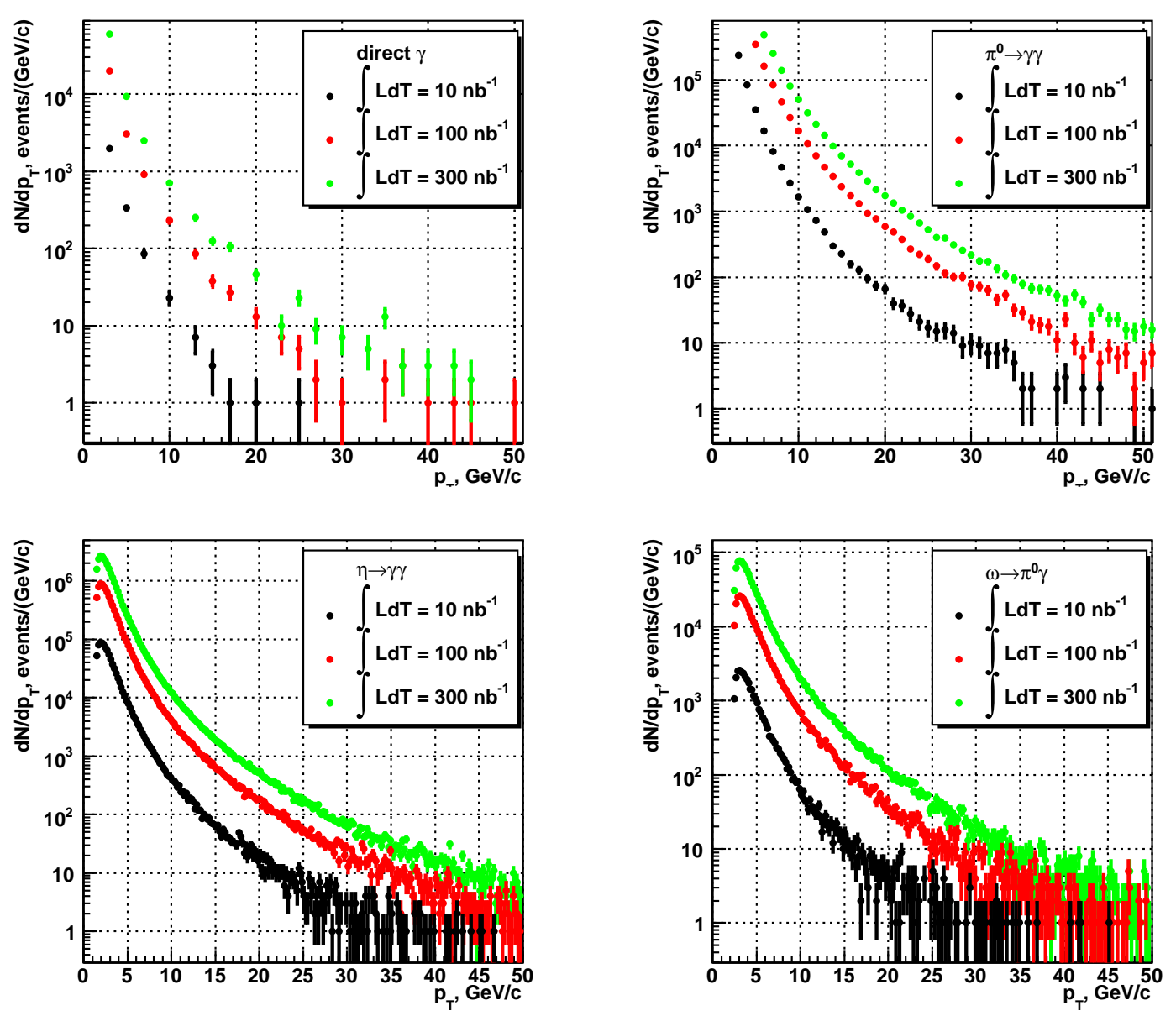

Figure 5: Number of detected direct photons and neutral mesons in 3 PHOS modules in $p p$ collisions at $\sqrt{s}=10 \mathrm{TeV}$ for $\int \mathscr{L} d T=10,100,300 \mathrm{nb}^{-1}$.

\section{References}

[1] ALICE collaboration. ALICE Technical Proposal. CERN/LHCC 95-71. 15 December 1995.

[2] ALICE collaboration. Photon Spectrometer PHOS, Technical Design Report. CERN/LHCC 99-4, 5 March 1999.

[3] ALICE collaboration. The electromagnetic calorimeter, Addendum to the Technical Proposal. CERN/LHCC 2006-014, 14 April 2006.

[4] ALICE collaboration. Technical Design Report of the Photon Multiplicity Detector (PMD). CERN/LHCC 99-32, 30 September 1999.

[5] ALICE Collaboration et al. J. Phys. G: Nucl. Part. Phys. 32 (2006) 1295-2040.

[6] A. Adare et al. [PHENIX Collaboration], Phys. Rev. Lett. 101 (2008) 232301 [arXiv:0801.4020 [nucl-ex]].

[7] F.Arleo et al., Photon physics in heavy ion collisions at the LHC. hep-ph/0311131. In: Hard Probes in Heavy Ion Collisions at the LHC Book, pages 367-493.

[8] P. Aurenche, M. Fontannaz, J. P. Guillet, B. A. Kniehl and M. Werlen, Eur. Phys. J. C 13,347 (2000), http://wwwlapp.in2p3.fr/lapth/PHOX_FAMILY.

[9] T. Sjostrand, S. Mrenna and P. Skands, JHEP 0605 (2006) 026 [arXiv:hep-ph/0603175]. 